\section{Check for updates}

Cite this: Soft Matter, 2018 14,9243

Received 5th September 2018, Accepted 21st October 2018

DOI: 10.1039/c8sm01815d

rsc.li/soft-matter-journal

\title{
Alcohol induced gelation of TEMPO-oxidized cellulose nanofibril dispersions $\uparrow+$
}

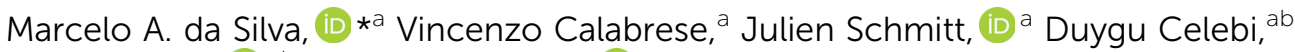 \\ Janet L. Scott (D) ab and Karen J. Edler (D)*a
}

\begin{abstract}
Solvent-induced physical hydrogels of TEMPO-oxidized cellulose nanofibrils (OCNFs) were obtained from aqueous/alcoholic dispersions of fibrils in lower alcohols, namely, methanol, ethanol, 1-propanol and 2-propanol. The sol-gel transition occurs above a critical alcohol concentration of ca. 30 wt\% for all alcohols tested. The rheological properties of the hydrogels depend on the nature of the alcohol: for ethanol, 1-propanol and 2-propanol the magnitude of the shear storage modulus follows the alcohol hydrophilicity, whilst methanol produces the weakest gels in the group. Above a second critical concentration, ca. 60 wt\% alcohol, phase separation is observed as the gels undergo syneresis. Analysis of small-angle X-ray scattering data shows that the OCNFs may be modelled as rigid rods. In the presence of lower alcohols, attractive interactions between nanofibrils are present and, above the alcohol concentration leading to gelation, an increase of the OCNF cross-section is observed, suggesting alcohol induced aggregation of nanofibrils.
\end{abstract}

\section{Introduction}

Self-assembly is a spontaneous natural process involving the formation of large complex structures from existing disordered building blocks, ${ }^{1,2}$ which may be directed by the intrinsic properties of the building blocks, e.g., shape and size, ${ }^{3-5}$ as well as specific or nonspecific attractive and repulsive interactions. ${ }^{6}$ Self-assembly is also influenced by the conditions and properties of the dispersing media, such as temperature, ${ }^{7}$ polarity, ${ }^{8}$ the presence of co-solutes, ${ }^{9}$ and preferential solvation. ${ }^{10}$ Selfassembling phenomena attract great academic interest with respect to understanding the spontaneous formation of complex assemblies, and technological interest as a tool to build designed materials with specific characteristics. In order to exploit the self-assembly process to build designed smart-materials in a "bottom-up" manner, an understanding of the interplay of these elements is necessary.

Cellulose-based materials offer a convenient source of renewable and cost-effective ingredients. By its very nature cellulose is renewable, sustainable and eco-friendly. ${ }^{11}$ Among the cellulose-based materials, nanocelluloses have shown great

\footnotetext{
${ }^{a}$ Department of Chemistry, University of Bath, Claverton Down, Bath, BA2 7AY, UK. E-mail: m.alves.da.silva@bath.ac.uk,k.edler@bath.ac.uk

${ }^{b}$ Centre for Sustainable Chemical Technologies, University of Bath, Claverton Down, Bath, BA2 7AY, UK

$\dagger$ Data sets supporting this article have been made freely available via the University of Bath Research Data Archive system at DOI: 10.15125/BATH-00568. \# Electronic supplementary information (ESI) available. See DOI: 10.1039/ c8sm01815d
}

potential as self-assembling materials. ${ }^{12-14}$ Nanocelluloses can be roughly divided into: cellulose nanofibrils (CNF), obtained via mechanical disintegration of wood pulp, hemp and flax, amongst other plant sources; cellulose nanocrystals (CNC), obtained from acid hydrolysis of cellulose fibrils; and bacterial nanocellulose (BNC), produced by bacteria. ${ }^{12}$

Cellulose nanofibrils have lengths of a few hundred nanometres and cross-sections of up to tens of nanometres, leading to particles with very large aspect ratios. ${ }^{15-17}$ The ease with which cellulose-based particles can be surface modified offers additional advantages, as particles can be tailored for specific applications. ${ }^{18,19}$ TEMPO-mediated oxidation ${ }^{20}$ is a chemoselective oxidation of the glucosyl C6 primary hydroxyl groups by $\mathrm{NaOCl}$ mediated by (2,2,6,6-tetramethyl-piperidin-1-yl)oxyl (TEMPO)/NaBr in water. These oxidized cellulose nanofibrils (OCNFs) are anionic particles and form stable dispersions of individualized nanofibrils in water. ${ }^{20-22}$ OCNFs can selfassemble in an aqueous environment due to the influence of concentration, ${ }^{23}$ OCNF aspect ratio, ${ }^{24}$ co-solutes, such as surfactants ${ }^{16}$ salts, ${ }^{9,25,26}$ or block copolymers ${ }^{27}$ as well as $\mathrm{pH}^{28-30}$ This flexibility makes OCNFs a good choice as building blocks in self-assembled hydrogels.

In this work, we studied the influence of low molecular weight alcohols on the self-assembly of OCNF dispersions. Above a critical alcohol concentration, OCNFs undergo gelation and eventual phase separation and this phenomenon can be related to nanoscale structural changes. Lower alcohols, such as ethanol or propanol, are commonly used in aqueous formulations, either to impart specific effects, e.g. ethanol in sterilising 
hand-cleansers, or to affect the solubilisation of active ingredients, which would not be soluble in water alone, thus this fundamental study also serves to enhance the understanding of the use of OCNFs as a formulation ingredient and rheology modifier.

\section{Experimental}

\section{Materials}

TEMPO oxidized cellulose nanofibrils, OCNFs, $\sim 25 \%$ degree of oxidation, produced from purified softwood fibre processed via high pressure homogenization, were kindly provided by Croda $^{\mathbb{B}}$. These were further purified by dialysis against ultra-pure water (DI water, $18.2 \mathrm{M} \Omega \mathrm{cm}$ ) and stirred at room temperature for $30 \mathrm{~min}$. Then the dispersion was acidified to $\mathrm{pH} 3$ using $\mathrm{HCl}$ solution and dialysed against ultra-pure water (cellulose dialysis tubing MWCO 12400) for 3 days with the DI water replaced twice daily. The dialysed OCNF suspension was processed via mechanical shear (ULTRA TURRAX, IKA T25 digital, 30 minutes at $6500 \mathrm{rpm}$ ) and the $\mathrm{pH}$ was adjusted to 7 using $\mathrm{NaOH}$ solution. This leads to the formation of a sodium-salt, as all - $\mathrm{COOH}$ groups on the OCNFs are now converted to COONa. After a second dialysis step the dispersion was diluted to $c a .2 \mathrm{wt} \%$ and dispersed using a sonication probe (Ultrasonic Processor, FB-505, Fisher), via a series of $1 \mathrm{~s}$ on $1 \mathrm{~s}$ off pulses for a net time of $5 \mathrm{~min}$ at $60 \%$ amplitude $(c a .300 \mathrm{~W})$ in an ice bath. The resultant material was concentrated to $3.0 \mathrm{wt} \%$ by the removal of solvent on a rotary evaporator at $60{ }^{\circ} \mathrm{C}$ and $10 \mathrm{mmHg}$ for approximately 2 hours. Methanol, ethanol, and 1- and 2-propanol (all $\geq 99.8 \%$ purity) were obtained from Sigma-Aldrich UK and used as provided. Ultra-pure water, resistivity $18.2 \mathrm{M} \Omega \mathrm{cm}$, was used throughout.

\section{Methods}

All samples were prepared by dilution of OCNF aqueous stock dispersions and concentrations are weight/weight.

Rheological measurements were conducted on a stresscontrolled Discovery Hybrid Rheometer, Model HR-3 (TA Instruments, USA) equipped with a crosshatched $20 \mathrm{~mm}$ parallel plate geometry over a sandblasted lower plate. Temperature was controlled via a Peltier unit $\left( \pm 0.1^{\circ} \mathrm{C}\right)$. A thin layer of low viscosity silicone-oil was added to the edge of the geometry to prevent evaporation. Oscillatory frequency sweeps were conducted at a strain $(\gamma)$ of $0.5 \%$ covering the angular frequency $(\omega)$ of 50 to $0.01 \mathrm{rad} \mathrm{s}^{-1}$. Flow curves were measured from 0.01 to $10 \mathrm{~s}^{-1}$. All experiments were conducted at $25{ }^{\circ} \mathrm{C}$.

Scanning Electron Microscope (SEM) images of cellulose aqueous and alcoholic dispersions (0.8 wt\%) were obtained using a JEOL JSM6480LV SEM (Jeol, USA) at $10 \mathrm{kV}$. Aqueous dispersions were freeze-dried, mounted on adhesive carbon tape and gold sputter-coated prior to imaging of the fibrils. The alcohol dispersions in methanol (100\%) or ethanol $(100 \%)$, prepared by solvent exchange, were sliced into $2 \times 2 \mathrm{~cm}$ squares, critical point dried from the alcohol and sputtercoated with gold.

Small-angle X-ray scattering (SAXS) measurements were conducted at the Diamond Light Source beamline I22 using a
PILATUS $2 \mathrm{M}$ (Dectris, Switzerland) detector. The X-ray wavelength used was $1 \AA$, corresponding to an energy of $12.4 \mathrm{keV}$, and the accessible $q$-range was 0.006 to $0.6 \AA^{-1}$. Data were reduced, and solvent and capillary contributions were subtracted, using the Dawn software. ${ }^{31}$ Experiments were conducted at room temperature. All data were fitted using internally developed routines written in FORTRAN. The data were fitted using a model of interacting rigid cylinders with elliptical cross sections and uniform scattering length density. ${ }^{32,33}$ The fitting parameters for the form factor of the fibrils are the major and minor radii of the cross-section of the cylinders, $R_{\max }$ and $R_{\min }$, respectively, and the length of the fibrils, $L$. Polydispersity of the cross section was not modelled. The effect of interactions on the scattering was calculated using the PRISM model, ${ }^{34}$ with the parameter $\nu_{\mathrm{RPA}}$, proportional to the concentration of cylinders, describing the strength of the interactions between the cylinders (the local excluded volume radius around each site being neglected for weak interactions). A detailed description of this model and its use for modelling cellulose based gels has been published by Schmitt et al. ${ }^{35}$

\section{Results and discussion}

Aqueous OCNF dispersions containing four low molecular weight alcohols, methanol, ethanol and 1- and 2-propanol, were studied. Mechanical behaviour was probed by both smallamplitude oscillatory (SAO) and flow shear rheology. SAO shear rheology offers insights into the quiescent structure of the dispersions, which enables the sol-gel transition to be probed on a macroscopic scale. The four alcohols tested resulted in similar rheological behaviour regarding the sol-gel transition, thus the figures presented focus on ethanol/water systems, with data pertaining to other alcohols contained in the ESI. $\$$ Two main behaviours can be readily observed with the increase of ethanol content: the storage modulus $\left(G^{\prime}\right)$ increases with the alcohol content and becomes less frequency dependent, indicated by the shallower slope of the curves, as illustrated for ethanol in Fig. 1A and for the other alcohols in the ESI, Fig. S1. The change in frequency dependence marks the change from a dispersion to a physical gel, reflecting the transition from a finite relaxation time to an infinite relaxation time (completely flat curve, $G^{\prime}$ constant). Generally, hydrogels can be divided into two groups: physical and chemical gels. The former are maintained by transient interactions, the latter by chemical bonds. For physical gels, $G^{\prime}$ will generally show some frequency dependence due to the dynamic nature of the transient interactions in contrast to the more static character of covalent bonding in a chemical gel. ${ }^{36}$

The increase of $G^{\prime}$ with increased alcohol concentration marks the progressive increase of the "stiffness" of the system, which can be seen as an increase of the connectedness of the OCNF in the dispersion. This could arise both from the formation of a connected network of fibrils or loss of mobility due to an increase of excluded volume. The variation of $G^{\prime}$ is not linear, as a sudden increase can be observed at around 30-40 wt\% depending on the alcohol (ESI, Fig. S2). The 

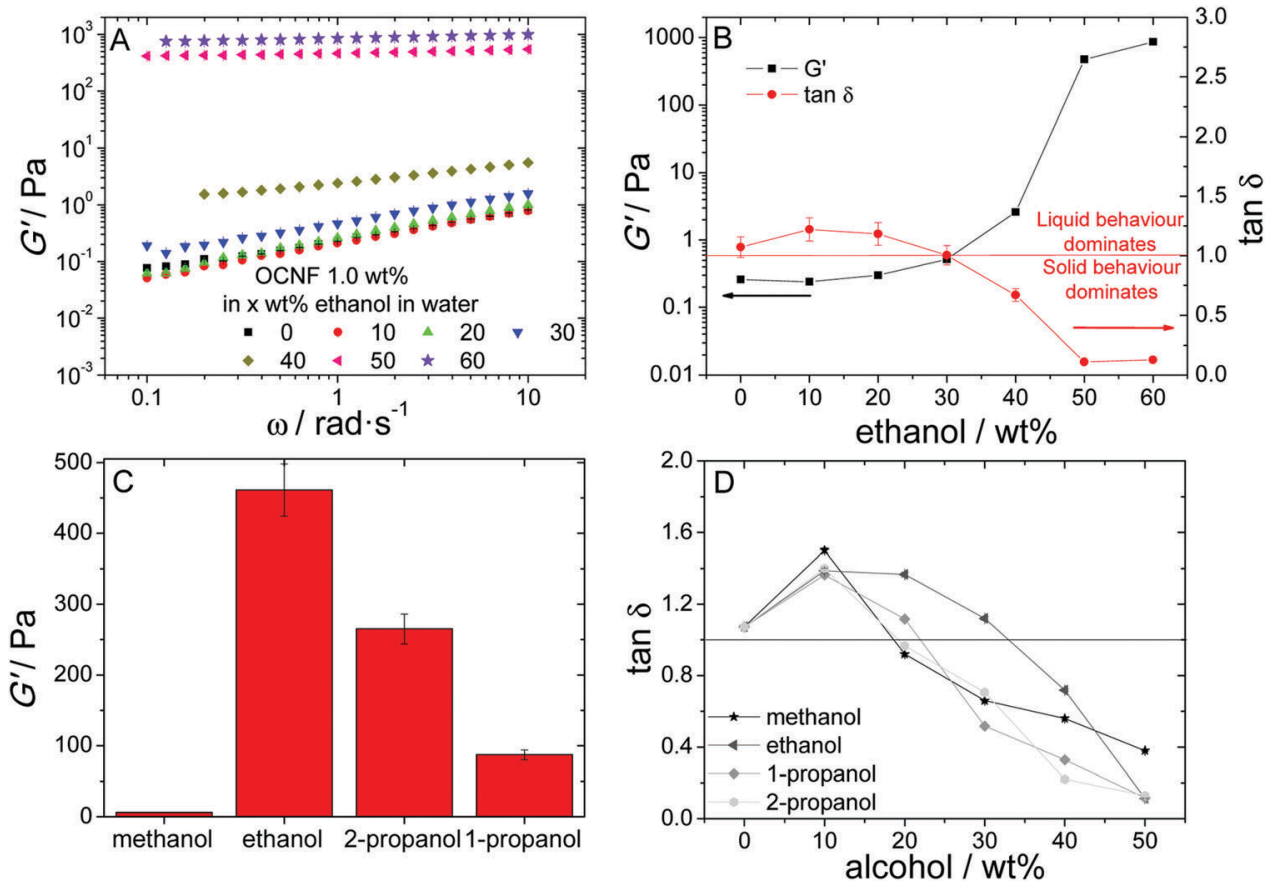

Fig. 1 (A) Oscillatory frequency sweeps for OCNF dispersions in water/ethanol mixtures as a function of ethanol concentration at a fixed OCNF content of $1.0 \mathrm{wt} \%$. (B) Dependence of $G^{\prime}(\mathbf{\square})$ and $\tan \delta(O)$ on the ethanol content of OCNF aqueous ethanol dispersions at $6.28 \mathrm{rad} \mathrm{s}^{-1}$. (C) Shear storage modulus $\left(G^{\prime}\right)$ values for OCNF hydrogels at $50 \mathrm{wt} \%$ of alcohol at $6.28 \mathrm{rad} \mathrm{s}^{-1}$. (D) Dependence of $\tan \delta$ on the alcohol content in OCNF dispersions at $6.28 \mathrm{rad} \mathrm{s}{ }^{-1}$.

tangent of the loss angle, $\delta$, is defined as the ratio of loss modulus to storage modulus $\left(\tan \delta=G^{\prime \prime} / G^{\prime}\right)$. Therefore, the parameter $\tan \delta$ indicates the balance between inelastic deformation, associated with the liquid-like behaviour, and elastic deformation, associated with solid-like behaviour. Thus, it can be used as an indicator of the gelation threshold, as $\tan \delta<1$ implies dominance of solid-like behaviour. This critical change in $G^{\prime}$ observed is also followed by a critical change in $\tan \delta$, and marks the gelation threshold for each alcohol (Fig. 1D).

Of the four low molecular weight alcohols studied, at $50 \mathrm{wt} \%$ of alcohol, methanol produces the weakest hydrogels, in terms of $G^{\prime}$ values, while ethanol yields the strongest, with 2-propanol exhibiting the second highest $G^{\prime}$ values (Fig. 1C). The capacity for inducing gelation, however, does not correlate with the hydrogel gel strength. Methanol and 1- and 2-propanol show rather similar behaviour (Fig. 1D), where $\tan \delta$ becomes $<1$ at around $20-30 \mathrm{wt} \%$ alcohol, whereas ethanol concentration must increase above $30 \mathrm{wt} \%$ to yield $\tan \delta$ values $<1$. Excluding methanol, a correlation can be established between the $G^{\prime}$ values measured for the hydrogels and alcohol hydrophobicity, as described by the 1-octanol/water partition coefficient $\left(\log P_{\text {ow }}\right)$, which increases in the order methanol $<$ ethanol $<2$-propanol $<1$-propanol ${ }^{37}$ (Table 1). The more hydrophilic alcohol (ethanol) produces the highest $G^{\prime}$ value, while the most hydrophobic one (1-propanol) produces gels with the lowest $G^{\prime}$ value (Table 1). Methanol, however, deviates from this trend: it is the most hydrophilic alcohol yet produces the weakest hydrogels.

To probe the effect of OCNF concentration on the gel properties, the alcohol concentration was kept constant at $50 \mathrm{wt} \%$ and the OCNF concentration varied from 0.4 to $1.2 \mathrm{wt} \%$. The frequency sweeps obtained are shown in Fig. 2A. For this con-
Table $1 \log P_{\text {ow }}$ values for the alcohols tested and $G^{\prime}$ and alcohol concentration at the gel point for OCNF alcohol dispersions

\begin{tabular}{lccl}
\hline & $\log P_{\mathrm{ow}}{ }^{37}$ & \multicolumn{1}{c}{${ }^{\prime a} / \mathrm{Pa}$} & Gelation concentration $\mathrm{wt}^{b}$ \\
\hline Methanol & -0.77 & $6.0 \pm 5$ & $18 \pm 2$ \\
Ethanol & -0.31 & $461 \pm 37$ & $33 \pm 3$ \\
2-Propanol & 0.05 & $265 \pm 21$ & $19 \pm 2$ \\
1-Propanol & 0.25 & $87 \pm 7$ & $22 \pm 2$
\end{tabular}

${ }^{a} G^{\prime}$ values for OCNF water/alcohol dispersions at $50 \mathrm{wt} \%$ alcohol content. ${ }^{b}$ Alcohol concentration required to gel OCNF water/alcohol dispersions.

centration range, all the samples studied showed $\tan \delta<1$ (Fig. 2B) reflecting a dominance of solid-like behaviour in all dispersions to OCNF concentrations as low as $0.4 \mathrm{wt} \%$. $G^{\prime}$ values show rapid growth as a function of the OCNF concentration for all alcohols studied up to $1.0 \mathrm{wt} \%$ (Fig. 2C); at $1.2 \mathrm{wt} \%$, methanol still shows a $G^{\prime}$ increase, however, $G^{\prime}$ drops for ethanol and plateaus for both 1-propanol and 2-propanol (Fig. 2C).

Flow behaviour, unlike SAO rheology, does not preserve the quiescent state. In this mode, the network that sustains the gel is disrupted to an extent that is related to the shear applied. The dependence of apparent viscosity $(\eta)$ versus shear rate $(\dot{\gamma})$ is presented in Fig. 3 for ethanol and in the ESI, $\$$ Fig. S4, for the other alcohols. Both aqueous and aqueous/alcoholic dispersions of OCNFs are shear-thinning. The dispersions show similar $\eta$ values at higher shear, but this high shear $\eta$ starts to diverge for different alcohols at concentrations above $30 \mathrm{wt} \%$, suggesting the presence of larger objects in the dispersions with higher alcohol content, even after the gel breakdown. Thus, it appears that elevated alcohol concentrations lead to OCNF aggregation. 

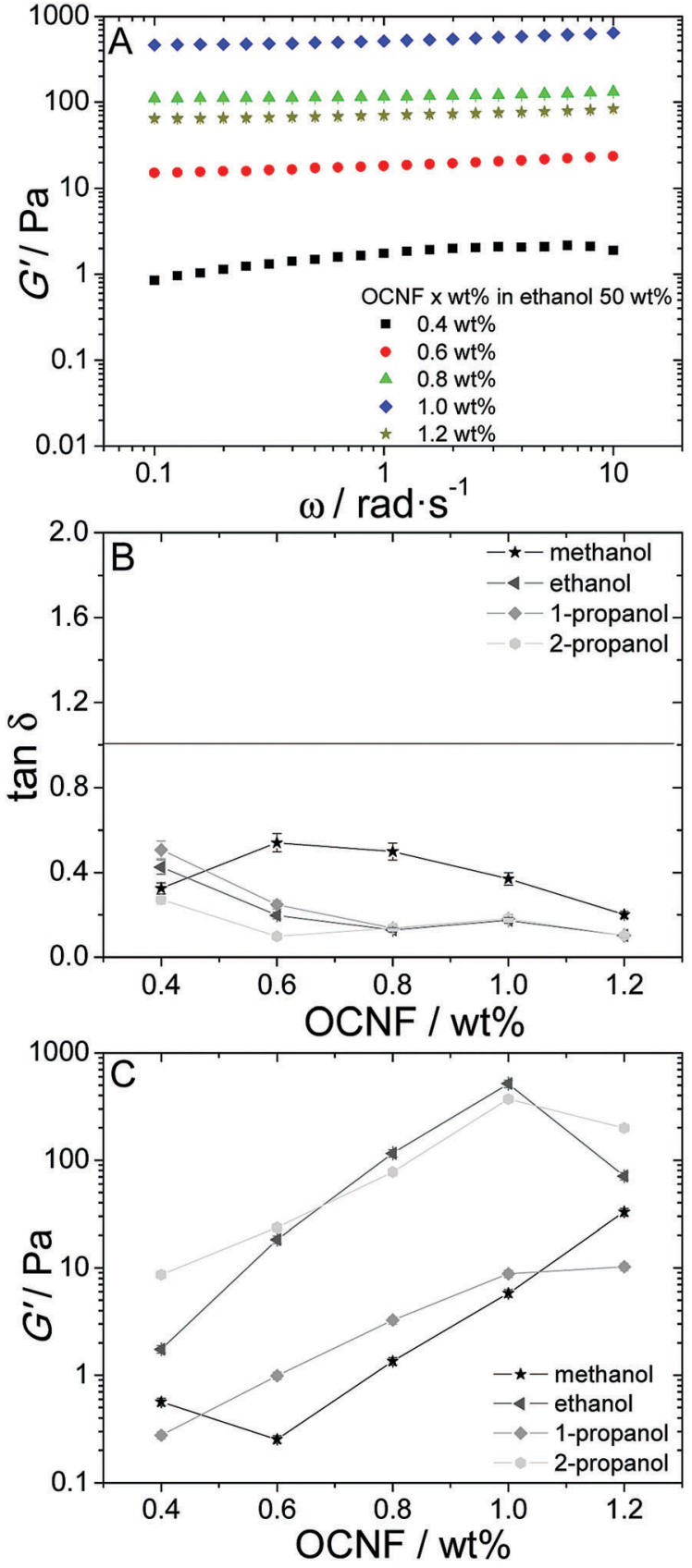

Fig. 2 (A) Oscillatory frequency sweeps for OCNF dispersions in water/ ethanol mixtures as a function of OCNF concentration and with a fixed ethanol content of $50 \mathrm{wt} \%$. Dependence of (B) $\tan \delta$ and (C) $G^{\prime}$ on the OCNF concentration for the four alcohols studied at $6.28 \mathrm{rad} \mathrm{s}^{-1}$.

Another factor suggesting alcohol-induced aggregation of the OCNFs is the significant syneresis observed: at alcohol concentrations of up to $50 \mathrm{wt} \%$, physical gels, stable over several weeks, are obtained, but increasing the alcohol concentration to $60 \mathrm{wt} \%$ and above leads to phase separation into a gel and syneresis fluid (for all except methanol) in a relatively short period of time, of the order of tens of minutes.

SEM images (Fig. 4) reflect the aggregation-inducing ability of the alcohols. Fig. 4A shows the freeze-dried product of a cellulose dispersion in water, where the randomly dispersed

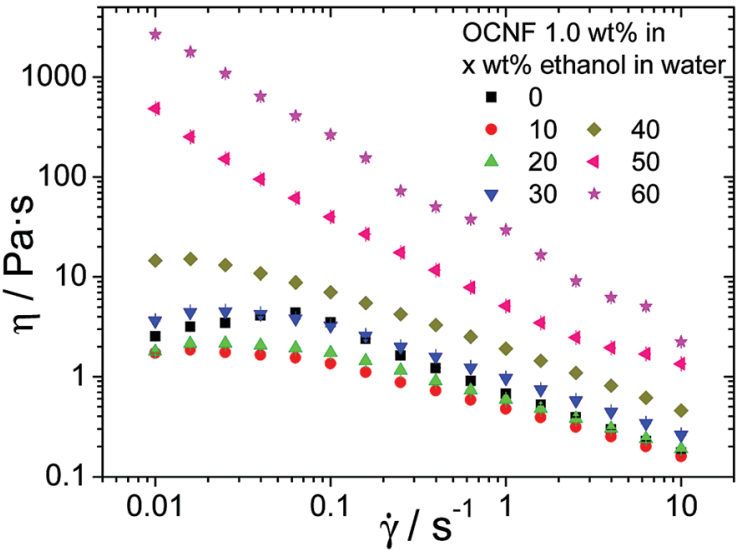

Fig. 3 Flow curves for OCNF dispersions in water/ethanol mixtures as a function of alcohol concentration and a fixed OCNF content of $1.0 \mathrm{wt} \%$.

fibrils form bundles in an isotropic network. Fig. 4B and C show supercritically dried methanol and ethanol OCNF dispersions, respectively. In both cases, a sheet like structure can be observed and the fibrous structure (Fig. 4A) is no longer distinguishable. The sheet-like structures observed in Fig. 4B and $\mathrm{C}$ have been described previously for nanocellulose films ${ }^{38}$ produced either from solvent-drying or filtration-based processes. ${ }^{39}$ This suggests that OCNFs have a tendency to aggregate into layered,

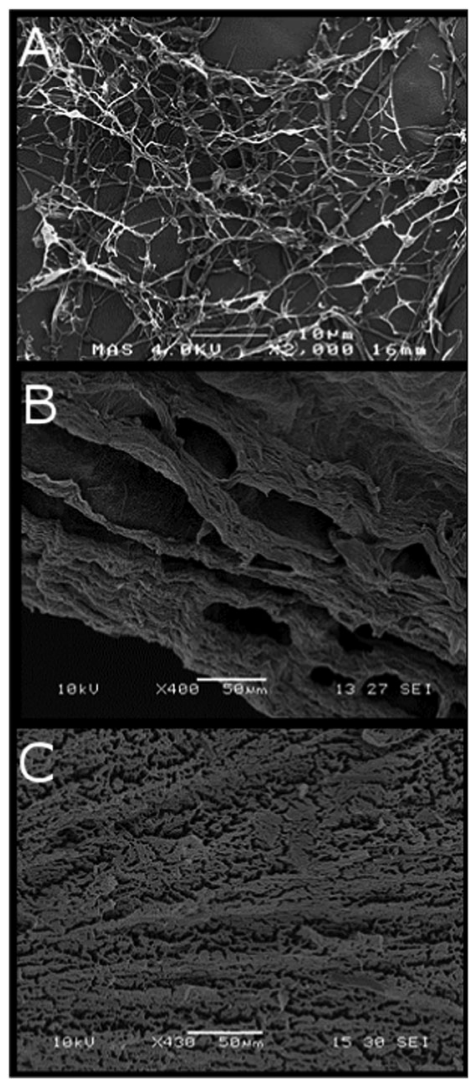

Fig. 4 SEM images of $0.8 w t \%$ OCNF dispersions (A) freeze dried from aqueous suspension, (B) supercritically dried from methanol, and (C) supercritically dried from ethanol. 
axially oriented structures, possibly reflecting the influence of the large aspect ratio of OCNFs as well as the effect of particle/ particle interactions arising with changes in the solvent, as described here.

The presence of fibril aggregates at lower alcohol concentrations was probed using small-angle X-ray scattering (SAXS) experiments. SAXS covers a length-scale window from several angstroms to $1000 \AA$, which is shorter than the expected longest
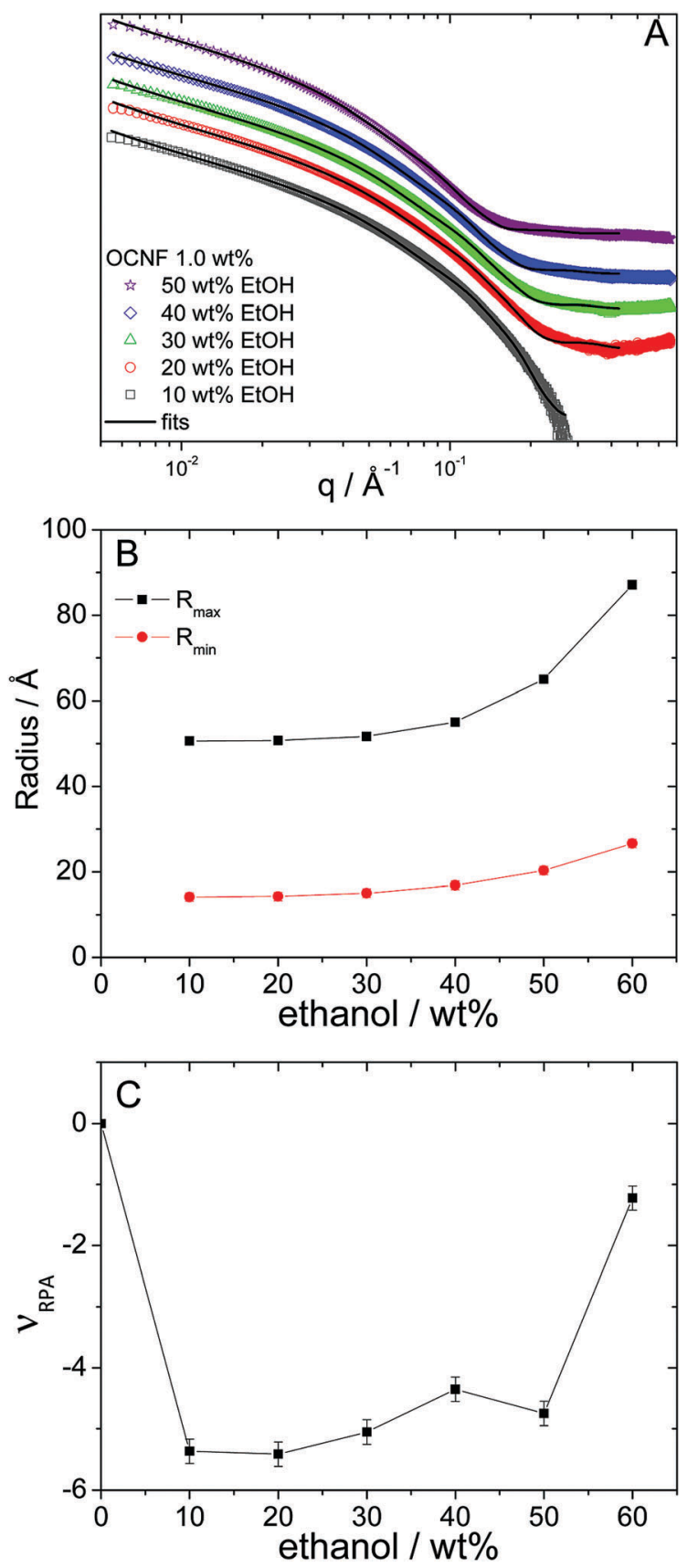

Fig. 5 (A) SAXS curves and fits for OCNF dispersions in water/ethanol mixtures as a function of ethanol concentration at a fixed OCNF content of $1.0 \mathrm{wt} \%$. Curves have been shifted for clarity. (B) Dependence of $R_{\max }$ ( and $R_{\min }(\mathcal{)})$ on ethanol concentration. (C) Dependence of $\nu_{\mathrm{RPA}}$ on ethanol concentration.
Table 2 Interaction parameter, $\nu_{\mathrm{RPA}}$, and major and minor radii obtained from fitting SAXS data using the interacting stiff rod model for OCNF water/alcohol dispersions at 10 and $50 \mathrm{wt} \%$ alcohol content

\begin{tabular}{llrl}
\hline & \multicolumn{1}{l}{$\nu_{\mathrm{RPA}}$} & $R_{\max } / \AA$ & $R_{\min } / \AA$ \\
\hline 10 wt\% alcohol & & & \\
Methanol & $-2.3 \pm-0.2$ & $55 \pm 1$ & $14 \pm 1$ \\
Ethanol & $-5.4 \pm-0.2$ & $51 \pm 1$ & $14 \pm 1$ \\
2-Propanol & 0.0 (fixed) & $64 \pm 1$ & $14 \pm 1$ \\
1-Propanol & $-4.9 \pm-0.2$ & $52 \pm 1$ & $14 \pm 1$ \\
& & & \\
50 wt\% alcohol & & $54 \pm 1$ & $17 \pm 1$ \\
Methanol & $-2.7 \pm-0.2$ & $65 \pm 1$ & $20 \pm 1$ \\
Ethanol & $-4.7 \pm-0.2$ & $68 \pm 1$ & $18 \pm 1$ \\
2-Propanol & $-4.8 \pm-0.2$ & $102 \pm 1$ & $24 \pm 1$ \\
1-Propanol & $-0.2 \pm-0.2$ & &
\end{tabular}

dimension of OCNFs (thousands of $\AA$ ), but within the lengthscale of the fibril cross-section (several $\AA$ ). ${ }^{17}$ SAXS data and curves arising from fitting of OCNF dispersions in water/ethanol mixtures at various ethanol concentrations are shown in Fig. 5A, and those of the other alcohols in the ESI, $\$$ Fig. S4. The model used to fit the data was that of interacting rigid-cylinders (better described as rods, given the aspect ratio of these fibrils) with ellipsoidal cross-sections. As the $q$-range does not allow the total length, $L$, of the fibrils to be probed, $L$ was fixed at $1600 \AA$, based on the dimensions measured from TEM images. It is worth noting that the choice of fixed fibril length will influence the absolute value of the parameter $\nu_{\mathrm{RPA}}$, but will not alter the relative values, which reflect the presence, and sign, of interaction. ${ }^{35}$ Both ellipsoidal radii values depend on the alcohol content and follow similar trends to the macroscopic properties (Fig. 5B and ESI, $\$$ Fig. S5), i.e. little dependency up to $40 \mathrm{wt} \%$ alcohol content, followed by a large increase of both radii at $50 \mathrm{wt} \%$ for ethanol and 1- and 2-propanol, with dispersions in methanol exhibiting little change (Table 2).

This apparent radial growth of the fibril dimensions signals a level of aggregation of OCNFs. This can also be correlated with the level of interactions between OCNFs, which can be modelled by the random phase approximation structure factor, $v i a$ the interaction term $\nu_{\mathrm{RPA}}$. These interactions are reflected in the changes in the slope of the signal in the low angle region. Non-interacting rods present a $q^{-1}$ slope associated with their rod-like structure: ${ }^{35}$ repulsive interactions would result in the lowering of the slope and attractive interactions (which could lead to aggregation) result in an increase in the slope. The interaction component of the model found by fitting the SAXS data suggests that fibril aggregation occurs, since a change from noninteracting OCNFs $\left(\nu_{\mathrm{RPA}}=0\right.$ for OCNFs at $1 \mathrm{wt} \%$ in water $)$ to attractive interactions between OCNFs $\left(\nu_{\mathrm{RPA}}<0\right)$ is found when alcohol is present (Table 2). It can be noted that for 2-propanol, fits of patterns at 10 and $20 \mathrm{wt} \%$ showed no interactions between fibrils ( $\nu_{\mathrm{RPA}}$ fixed at 0$)$. Nonetheless, one can note that for this particular alcohol, the signal at small-angle shows a strong increase (see the ESI, $\$$ Fig. S4C), which was modelled by a Porod signal $I_{\text {Porod }}(q)=K / q^{4}$, with $K$ being a constant. This extra signal is used to describe large aggregates in solution with a smooth interface, possibly from impurities in the solution, and influence 
the extraction of the interaction parameter $\nu_{\mathrm{RPA}}$. At $60 \mathrm{wt} \%$ alcohol content, where syneresis starts to manifest, large increases in the apparent fibril radii (Fig. 5B and ESI, + Fig. S5) and decreases in the values of the interaction parameter (Fig. 5C and ESI, $\$$ Fig. S6) suggest extensive aggregation of OCNFs, in agreement with the observation that macroscopic phase separation (syneresis) occurs. For 1-propanol, the most hydrophobic alcohol of this series, this decrease of the interaction parameter can already be detected at $50 \mathrm{wt} \%$, highlighting the effect of the alcohol hydrophobicity on the gelation process.

In summary, a sol-gel transition of OCNF dispersions can be induced by the presence of an alcohol co-solvent. The addition of alcohol leads to an increase of the dispersion viscosity up to a critical concentration, $c a .30 \mathrm{wt} \%$, after which a sudden increase in the values of $G^{\prime}$ and drop in the values of $\tan \delta$ are observed, related to a gelation threshold. A further increase of alcohol content, to above $c a$. $60 \mathrm{wt} \%$, eventually leads to syneresis. This suggests self-aggregation of OCNFs, indicating a point where the particle-to-particle attractive interactions lead to a compaction of the gel, expelling solvent from the gel network. SAXS data support the aggregation of OCNFs triggered by the presence of alcohols, as the data collected show a slow increase in the apparent OCNF cross-section size, followed by a sudden increase at the gelation concentration (except for methanol). Furthermore, the SAXS data can be fitted with a rigid rod model at alcohol concentrations of up to $50 \mathrm{wt} \%$, but this model no longer accurately reproduces the SAXS patterns at higher alcohol concentrations. This evidence for aggregation is in agreement with flow curves that show that, above the critical concentration, larger aggregates are likely to be present in the dispersions.

This behaviour is analogous, at least in part, to the lower solubility of $\mathrm{NaCl}$ in alcohols. OCNF dispersions are stable due to the electrostatic repulsion from the -COONa groups (as OCNFs are in the sodium salt form), which are fully dissociated in water. The zeta-potential of an OCNF dispersion at $1 \mathrm{wt} \%$ is ca. $-60 \mathrm{mV}$. As the alcohol content increases in the water/ alcohol dispersions, the solubility of the sodium ion in the mixed solvent also decreases. At $40 \mathrm{wt} \%$ alcohol, the $\mathrm{NaCl}$ solubility is ca. $13 \mathrm{wt} \%$ in methanol and $12 \mathrm{wt} \%$ in ethanol, which is half the solubility in pure water, $27 \mathrm{wt} \%$. At $60 \mathrm{wt} \%$ ethanol, sodium chloride has only one quarter of its solubility in pure water; 7.2 and $6.0 \mathrm{wt} \%$ for methanol and ethanol, respectively. ${ }^{40}$ Assuming that sodium ions from OCNFs follow the same trend as in $\mathrm{NaCl}$, the condensation of the $\mathrm{COO}^{-} \mathrm{Na}^{+}$ion pair will lead to a reduction in the width of the electrical double-layer, which in turn, favours aggregation of OCNFs. However, the difference in the solubility of $\mathrm{NaCl}$ in ethanol and methanol is not large enough to explain the differences observed between these alcohols, with methanol-induced gels being much weaker than ethanol-induced gels (Fig. 1C). This suggests that other mechanisms must also be involved. A similar gelation behaviour upon addition of low molecular weight alcohols has been reported for clay platelet dispersions in water, where the clay particles are also in the sodium-salt form. ${ }^{10}$ This suggests that this phenomenon is applicable to a range of dispersions of surface charged (nano)particles with large aspect ratios.
Kimura and Haraguchi suggested that gelation in the clay platelet system was driven by the presence of water-rich and alcohol-rich regions present in the water/alcohol mixture, i.e., the formation of microdomains. ${ }^{10}$ Self-association of water and alcohol molecules in alcoholic aqueous binary mixtures leading to the occurrence of inhomogeneous clustering at short length scales has been suggested in the literature. ${ }^{41-44}$ Dixit et al. ${ }^{41}$ used neutron diffraction to provide evidence for incomplete mixing at the molecular level in methanol/water mixtures. Wakisaka et al. ${ }^{42,43}$ used a specially designed mass spectrometer to probe mixing in primary alcohol aqueous binary mixtures. They investigated molecular clustering in water-ethanol, water-1-propanol and water-1-butanol binary mixtures by analysing the mass spectra of clusters generated through the fragmentation of liquid droplets and suggested that the alcohols form microscale hydrophobic environments due to preferential self-association. This phenomenon is observed at a lower alcohol concentration in 1-propanol than ethanol. The formation of such micro-domains could lead to spatial segregation of OCNFs, which would concentrate in the diminishing water domains as the alcohol content increases and the extent of the aggregation would be tied to the ability of the alcohol to create these micro-domains. The presence of microheterogeneities in water/alcohol mixtures has been suggested as a trigger for gelation in various types of systems, such as proteins in solution, ${ }^{45,46}$ and it appears that this phenomenon may be more generally applicable. Other mechanisms could also be involved. For instance, van Uden et $a l^{47}$ observed that, for the pressure-driven gelation of potato starch grains, alcohol-water mixtures required higher pressure as mixtures have lower cohesive energy than the neat solvents, affecting the starch particle behaviour. An increase of solvent hydrophobicity, due to addition of co-solvents, is also proposed as a mechanism for gelation of polysaccharides, but this refers mostly to soluble macromolecules, instead of nanoparticles. ${ }^{48,49}$

\section{Conclusions}

In this work, solvent induced gelation of OCNF dispersions in water/alcohol mixtures (methanol, ethanol and 1- and 2-propanol) was investigated at the macro and nano-scales. Bulk rheology points to the ability of low molecular weight alcohols to trigger physical gelation of the OCNF dispersion above a certain critical alcohol concentration. While all four alcohols tested were able to induce gelation, the gel rheological properties depend on the nature of the alcohol: excluding methanol, the gel bulk rheological properties were correlated with the alcohol hydrophilicity, with the most hydrophilic alcohol studied, ethanol, producing the strongest gels and the most hydrophobic one, 1-propanol, producing the weakest gel. Information derived from fitting of SAXS data suggests that the gel formation is driven by aggregation of OCNFs, as the presence of alcohol increases interactions between nanofibrils, leading to a sudden increase in the apparent radii describing the OCNF crosssection, which correlates well with the critical concentration for gel formation and phase separation. 


\section{Conflicts of interest}

There are no conflicts to declare.

\section{Acknowledgements}

The authors thank EPRSC for funding this project (grant EP/N033310/1). V. Calabrese thanks the University of Bath for supporting his PhD. We thank the Diamond Light Source for access to beamline I22 (SM16364-1) that contributed to the results presented here, and we would like to thank Dr N. Terrill and Dr A. Smith for their assistance with the experiment on beamline I22.

\section{References}

1 G. M. Whitesides and B. Grzybowski, Science, 2002, 295, 2418-2421.

2 M. Grzelczak, J. Vermant, E. M. Furst and L. M. Liz-Marzán, ACS Nano, 2010, 4, 3591-3605.

3 S. Sacanna, D. J. Pine and G.-R. Yi, Soft Matter, 2013, 9, 8096-8106.

4 L. Wu, C. P. Ortiz and D. J. Jerolmack, Langmuir, 2017, 33, 622-629.

5 Q. Wu, Y. Meng, S. Wang, Y. Li, S. Fu, L. Ma and D. Harper, J. Appl. Polym. Sci., 2014, 131, 40525.

6 J. Israelachvili and M. Ruths, Langmuir, 2013, 29, 9605-9619.

7 S. Hocine and M.-H. Li, Soft Matter, 2013, 9, 5839-5861.

8 J. Kim, C.-H. Choi, S.-J. Yeom, N. Eom, K.-K. Kang and C.-S. Lee, Langmuir, 2017, 131, 7503-7511.

9 H. Dong, J. F. Snyder, K. S. Williams and J. W. Andzelm, Biomacromolecules, 2013, 14, 3338-3345.

10 Y. Kimura and K. Haraguchi, Langmuir, 2017, 33, 4758-4768.

11 D. Klemm, B. Heublein, H.-P. Fink and A. Bohn, Angew. Chem., Int. Ed., 2005, 44, 3358-3393.

12 D. Klemm, F. Kramer, S. Moritz, T. Lindström, M. Ankerfors, D. Gray and A. Dorris, Angew. Chem., Int. Ed., 2011, 50, 5438-5466.

13 S. J. Eichhorn, A. Dufresne, M. Aranguren, N. E. Marcovich, J. R. Capadona, S. J. Rowan, C. Weder, W. Thielemans, M. Roman, S. Renneckar, W. Gindl, S. Veigel, J. Keckes, H. Yano, K. Abe, M. Nogi, A. N. Nakagaito, A. Mangalam, J. Simonsen, A. S. Benight, A. Bismarck, L. A. Berglund and T. Peijs, J. Mater. Sci., 2009, 45, 1-33.

14 A. Isogai, J. Wood Sci., 2013, 59, 449-459.

15 O. Nechyporchuk, M. N. Belgacem and J. Bras, Ind. Crops Prod., 2016, 93, 2-25.

16 R. J. Crawford, K. J. Edler, S. Lindhoud, J. L. Scott and G. Unali, Green Chem., 2012, 14, 300-303.

17 O. Nechyporchuk, M. N. Belgacem and F. Pignon, Biomacromolecules, 2016, 17, 2311-2320.

18 S. Eyley and W. Thielemans, Nanoscale, 2014, 6, 7764-7779. 19 S. Kalia, S. Boufi, A. Celli and S. Kango, Colloid Polym. Sci., 2014, 292, 5-31.

20 T. Saito, S. Kimura, Y. Nishiyama and A. Isogai, Biomacromolecules, 2007, 8, 2485-2491.

21 A. Isogai, T. Saito and H. Fukuzumi, Nanoscale, 2011, 3, 71-85.
22 S. Montanari, M. Roumani, L. Heux and M. R. Vignon, Macromolecules, 2005, 38, 1665-1671.

23 L. Geng, N. Mittal, C. Zhan, F. Ansari, P. R. Sharma, X. Peng, B. S. Hsiao and L. D. Söderberg, Macromolecules, 2018, 51, 1498-1506.

24 M. Nordenström, A. Fall, G. Nyström and L. Wågberg, Langmuir, 2017, 33, 9772-9780.

25 H. Fukuzumi, R. Tanaka, T. Saito and A. Isogai, Cellulose, 2014, 21, 1553-1559.

26 A. Sone, T. Saito and A. Isogai, ACS Macro Lett., 2016, 5, 1402-1405.

27 T. Ingverud, E. Larsson, G. Hemmer, R. Rojas, M. Malkoch and A. Carlmark, J. Polym. Sci. A1, 2016, 54, 3415-3424.

28 T. Saito, T. Uematsu, S. Kimura, T. Enomae and A. Isogai, Soft Matter, 2011, 7, 8804-8809.

29 K. Abe and H. Yano, Carbohydr. Polym., 2011, 85, 733-737.

30 L. Mendoza, W. Batchelor, R. F. Tabor and G. Garnier, J. Colloid Interface Sci., 2018, 509, 39-46.

31 DawnScience, http://dawnsci.org/, Accessed 03/03/2017.

32 J. S. Pedersen and P. Schurtenberger, Macromolecules, 1996, 29, 7602-7612.

33 W.-R. Chen, P. D. Butler and L. J. Magid, Langmuir, 2006, 22, 6539-6548.

34 J. S. Pedersen, in Neutrons, X-rays, and light: scattering methods applied to soft condensed matter, ed. P. Lindner and T. Zemb, Elsevier, Amsterdam, Editon edn, 2002, vol. 391-420.

35 J. Schmitt, V. Calabrese, M. A. da Silva, S. Lindhoud, V. Alfredsson, J. L. Scott and K. J. Edler, Phys. Chem. Chem. Phys., 2018, 20, 16012-16020.

36 G. M. Kavanagh and S. B. Ross-Murphy, Prog. Polym. Sci., 1998, 23, 533-562.

37 F. P. Ballistreri, C. G. Fortuna, G. Musumarra, D. Pavone and S. Scire, ARKIVOC, 2002, XI, 54-64.

38 M. Henriksson, L. A. Berglund, P. Isaksson, T. Lindström and T. Nishino, Biomacromolecules, 2008, 9, 1579-1585.

39 F. Ansari and L. A. Berglund, Biomacromolecules, 2018, 19, 2341-2350.

40 S. P. Pinho and E. A. Macedo, Fluid Phase Equilib., 1996, 116, 209-216.

41 S. Dixit, J. Crain, W. C. K. Poon, J. L. Finney and A. K. Soper, Nature, 2002, 416, 829-832.

42 A. Wakisaka, S. Komatsu and Y. Usui, J. Mol. Liq., 2001, 90, 175-184.

43 A. Wakisaka and T. Ohki, Faraday Discuss., 2005, 129, 231-245. 44 Y. Marcus, Phys. Chem. Chem. Phys., 1999, 1, 2975-2983.

45 M. A. da Silva, O. A. El Seoud and E. P. G. Arêas, J. Mol. Struct., 2007, 841, 51-60.

46 W. S. Gosal, A. H. Clark and S. B. Ross-Murphy, Biomacromolecules, 2004, 5, 2430-2438.

47 N. W. A. van Uden, H. Hubel, D. A. Faux, A. C. Tanczos, B. Howlin and D. J. Dunstan, J. Phys.: Condens. Matter, 2003, 15, 1577-1584.

48 A. Demilecamps, C. Beauger, C. Hildenbrand, A. Rigacci and T. Budtova, Carbohydr. Polym., 2015, 122, 293-300.

49 G. Tkalec, Z. Knez and Z. Novak, RSC Adv., 2015, 5, 77362-77371. 